\title{
1 SARS-CoV2 Testing: The Limit of Detection Matters
}

3 Running Title: LoD Matters

5 Ramy Arnaout, ${ }^{a, c, f ~ \# ~ R o s e ~ A . ~ L e e, ~}{ }^{a, b, c}$ Ghee Rye Lee, ${ }^{e}$ Cody Callahan, ${ }^{d}$ Christina F. Yen, ${ }^{b, c}$

6 Kenneth P. Smith, ${ }^{a, b}$ Rohit Arora, ${ }^{a, c}$ James E. Kirby, ${ }^{a, c, \#}$

$7 \quad{ }^{a}$ Department of Pathology, Beth Israel Deaconess Medical Center, Boston, MA, USA

$8{ }^{b}$ Division of Infectious Diseases, Department of Medicine, Beth Israel Deaconess Medical

9 Center, Boston, MA, USA

$10{ }^{\mathrm{C}}$ Harvard Medical School, Boston, MA, USA

11 dDepartment of Radiology, Beth Israel Deaconess Medical Center, Boston, MA, USA 02215

12 eDepartment of Surgery, Beth Israel Deaconess Medical Center, Boston, MA, USA 02215

13 'Division of Clinical Informatics, Department of Medicine, Beth Israel Deaconess Medical Center,

14 Boston, MA USA 02215

15 \#Co-Corresponding Authors (order determined alphabetically)

16 Ramy Arnaout and James E. Kirby

17 Beth Israel Deaconess Medical Center

18330 Brookline Avenue - YA309

19 Boston, MA 02215

20 jekirby@bidmc.harvard.edu

21 Phone: 617-667-3648

22 Fax: 617-667-4533 


\section{Abstract}

26 Resolving the COVID-19 pandemic requires diagnostic testing to determine which individuals

27 are infected and which are not. The current gold standard is to perform RT-PCR on

28 nasopharyngeal samples. Best-in-class assays demonstrate a limit of detection (LoD) of 100

29 copies of viral RNA per milliliter of transport media. However, LoDs of currently approved

30 assays vary over 10,000-fold. Assays with higher LoDs will miss more infected patients,

31 resulting in more false negatives. However, the false-negative rate for a given LoD remains

32 unknown. Here we address this question using over 27,500 test results for patients from across

33 our healthcare network tested using the Abbott RealTime SARS-CoV-2 EUA. These results

34 suggest that each 10 -fold increase in $L O D$ is expected to increase the false negative rate by

$3513 \%$, missing an additional one in eight infected patients. The highest LoDs on the market will

36 miss a majority of infected patients, with false negative rates as high as $70 \%$. These results

37 suggest that choice of assay has meaningful clinical and epidemiological consequences. The

38 limit of detection matters. 


\section{Introduction}

41 In response to the SARS-CoV-2 pandemic being declared a public health emergency, clinical

42 and commercial laboratories as well as test kit manufacturers have been submitting diagnostic

43 devices and assays for expedited Emergency Use Authorization by the Food and Drug

44 Administration (FDA EUA). As of June 2020, there were over 85 such EUA issuances for

45 COVID-19 diagnostics (https://www.fda.gov/medical-devices/emergency-situations-medical-

46 devices/emergency-use-authorizations, accessed June 1, 2020). However, optimal use of these

47 assays requires consideration of several issues.

48 First, NP swabs are generally considered to provide optimal detection early in disease.

49 However, even for this sample type, there is currently no ideal reference standard to establish

50 clinical sensitivities of the available EUA SARS-CoV-2 diagnostic assays (1). Second, details

51 about assay limit of detection (LoD) are often not provided with sufficient detail and

52 transparency to allow facile comparisons. For molecular diagnostic assays, the LoD is generally

53 considered the lowest concentration of target that can be detected in $\geq 95 \%$ of repeat

54 measurements. The LoD is a measure of analytic sensitivity, as opposed to clinical sensitivity,

55 which measures the fraction of infected people detected by a given test. LoDs are sometimes

56 reported in units other than copies of viral genomic RNA per milliliter of transport media

57 (copies $/ \mathrm{mL}$ ), such as $\mathrm{TCID}_{50}$, copies/microliter, copies per reaction volume, or molarity of assay

58 target, making comparisons difficult. Third, the LoDs of currently approved EUA nucleic acid

59 amplification and antigen detection tests for SARS-CoV-2 vary up to 10,000 fold (see below)

60 and likely are associated with meaningful differences in clinical sensitivity for these tests.

61 Fourth, although LoDs are quantitative, and RT-PCR tests are inherently quantitative, in practice

62 results for SARS-CoV-2 testing are generally reported qualitatively, as positive or negative,

63 even though viral load may provide both clinically and epidemiologically important information. 
64 Two barriers to quantitative reporting are demonstration that qPCR cycle threshold (Ct) values

65 are repeatable with acceptably low variance and a reliable means of converting from Ct value to

66 viral load. The latter is complicated by a traditional requirement for a standard curve that must

67 span a range of viral loads at least as large as what is observed in the patient population, which

68 can be expensive and time-consuming, especially in a pandemic where the limits of this range

69 are unknown; however, there have been reports demonstrating how appropriate measurements,

70 based on the principles of RT-PCR, can be used as an alternative for reliable conversion of Ct

71 values to viral loads $(2,3)$.

72 Here we report on the reliability of Cts for the Abbott SARS-CoV-2 EUA (LoD 100 copies viral

$73 \mathrm{RNA} / \mathrm{mL}$ transport medium, among the best in class) (4) and a conversion from Ct to viral load,

74 which together support the use of reporting viral loads clinically, and also on an observation

75 based on over 4,700 first-time positive results that makes it possible to estimate the clinical

76 sensitivity and false-negative rate of both this assay and other assays that have received EUA

77 for detecting SARS-CoV-2 infection. These findings have clear implications for patient care,

78 epidemiology, and the social and economic management of the ongoing pandemic.

\section{Methods}

80 Setting and time period. All SARS-CoV-2 testing data from The Beth Israel Lahey Health

81 Network from March 26th to May 2nd, 2020 was included in our analysis. The study was

82 deemed exempt by our hospital institutional review board.

83 Testing. Tests were performed using the Abbott RealTime SARS-CoV-2 assay, a real-time

84 reverse transcriptase $(\mathrm{RT})$ polymerase chain reaction (PCR) test for qualitative detection of

85 SARS-CoV-2 in NP and oropharyngeal swabs (5). The dual target assay detects both the

86 SARS-CoV-2 RdRp and $\mathrm{N}$ genes with a reported LoD of 100 copies $/ \mathrm{mL}$. The assay also 
includes an internal control. Results are reported as positive if the Ct value is $\leq 31.5$, based upon

results were analyzed. Repeat tests were excluded in order to more accurately estimate the

90 range of $\mathrm{Ct}$ values of the infected population upon presentation at our medical center. In our

91 internal validation we determined that the LoD with $100 \%$ detection for the Abbott m2000

92 platform was 100 copies $/ \mathrm{mL}(\mathrm{n}=80)$, with $\mathrm{Ct}$ mean and standard deviation at this LoD,

$9326.06 \pm 1.03$ (4). Note, the Ct determination on Abbott M2000rt platform is alternatively called the

94 fractional cycle number (FCN) and is specifically one way of determining the cycle number at

95 the maximum amplification efficiency inflection point, i.e, the maxRatio, of each amplification

96 curve (6). The FCN has been reported to be a more robust measure for Ct determination than a

97 fixed fluorescence threshold.

98 Statistics. Variance was estimated by $R^{2}$ of $\mathrm{Ct}$ values for repeat tests obtained within 6 hours

99 ( $n=25$ patients, excluding one obvious outlier that by itself accounted for half the total variance:

100 initial Ct 4.4, but repeat negative and attributed to pre-analytic or analytic technical error) and 12

101 hours ( $n=51$ patients, excluding the same outlier). The conversion from $C t$ value to viral load

102 was performed using the definition of exponential growth with variable efficiency $(2,3)$.

103 Efficiency was measured from plots of fluorescence intensity vs. cycle number for 50 positive

104 samples chosen at random, yielding an expression for viral load in copies $/ \mathrm{mL}$ as a function of $\mathrm{Ct}$

105 (Eq. 6, Supplementary Methods). Per this expression, the expected negative cutoff corresponds

106 to 9.2 copies per $\mathrm{mL}$ or $\sim 2$ virions per RT-PCR reaction volume $(0.5 \mathrm{~mL})$, supporting the validity

107 of our parameter estimation.

108 We used Python (v3.6) and its NumPy, SciPy, Matplotlib, and Pandas libraries to plot linear

109 regression and Theil-Sen slopes with 95\% confidence intervals on repeat positives; a

110 normalized cumulative distribution (histogram) of positive results (with reversed $\mathrm{x}$-axis for ease 
111 of interpretation); binned histogram by 0.5 log10 units, and linear regression on log 10-

112 transformed data.

\section{Results}

114 Of the 27,098 tests performed on 20,076 patients over the testing period, 6,037 tests were 115 positive (22\%), representing 4,774 unique patients. Analysis of repeats within 6 or 12 hours of

116 each other (7) demonstrated high repeatability of Ct values over these short time windows $\left(R^{2}\right.$

1170.70 and $0.63, n=25$ and 51 , respectively), supporting the validity of this quantitative measure as

118 a basis for assessment of viral load in patients (Fig. 1). We used basic principles of PCR and

119 detailed measurements of PCR efficiency on 50 randomly chosen positive samples to convert

120 from Ct values to viral load, in units of copies of viral RNA per $\mathrm{mL}$ of viral transport medium. In

121 order to study the patient population upon presentation without confounding by repeat

122 measurements on the same patients, the remainder of the analysis was on the first positive

123 value for the above 4,774 unique patients.

124 Viral loads spanned nearly nine orders of magnitude, from 9 copies $/ \mathrm{mL}$ to 2.5 billion copies $/ \mathrm{mL}$

125 (Fig. 2). Notably, patients were almost equally likely to exhibit low, medium, or high viral loads

126 upon initial testing, with remarkable uniformity down to the LoD of 100 copies $/ \mathrm{mL}\left(R^{2}=0.99\right)$. The

127 reason for this uniformity is unknown. Fewer patients had viral loads below the LoD, as reflected

128 by the curve's departure from the trend in this range. Because the LoD is a $95 \%$ confidence

129 limit, the difference between the curve and the trend likely reflects false negatives: the lower the

130 viral load, the greater the likelihood that infection will be missed. By definition, only $5 \%$ of

131 patients with viral load at the LoD are expected to be missed ( 1 in 20 patients); this percentage

132 grows for patients with viral loads below this threshold. Thus, extending the observed trend

133 leftward to the assay's positive cutoff, which corresponds to approximately two virions per 
134 reaction, yields an estimate of the total false negative rate for this assay of $10 \%$, and thus a

135 clinical sensitivity of $90 \%$, or 9 in 10 infected individuals.

136 This method can be used to estimate the clinical sensitivity of assays with other LoDs. For

137 example, an assay with LoD of 1,000 copies/mL, such as that of the CDC assay (8) or Genmark

138 ePlex EUA (9), is expected to detect $77 \%$, or 3 in 4 , of infected individuals, for a false-negative

139 rate of $22 \%$. With an LoD of 6,250 copies/mL, the LabCorp COVID-19 RT-PCR EUA test has an

140 estimated clinical sensitivity of $67 \%$ and a false-negative rate of $33 \%$, missing approximately 1

141 in 3 infected individuals. The first EUA antigen detection assay, the Quidel Sofia2 SARS Antigen

142 FIA, has an LoD of approximately 6 million in a contrived universal transport medium sample

143 collection. Although the package insert indicates the LoD using TCID 50 units, the BEI Resources

144 control material referenced lists both $\mathrm{TCID}_{50}$ and genome copies $/ \mathrm{mL}$, allowing the calculation of

145 the latter and an associated estimated clinical sensitivity of $31 \%$, i.e., it would miss 7 in 10

146 infected patients.

\section{Discussion}

148 The diagnostic priorities in the COVID-19 pandemic are to robustly identify three populations:

149 the infected, the infectious, and the susceptible. Our study addresses the first of these.

150 Specifically, it illustrates the clinical and epidemiologic impact of assay LoD on SAR-CoV-2

151 diagnosis and the challenges of interpreting and comparing molecular assay results across

152 various platforms. First, viral loads vary widely among infected individuals, from individuals with

153 extremely high viral loads, potential "super-spreaders" who presumably would be picked up by

154 even the least sensitive assays, to those whose viral loads are near, at, or even below the LoD

155 of many assays. Therefore, a substantial fraction of infected patients will be missed by less

156 sensitive assays. Concerningly, some of these missed patients are, have been, or will become

157 infectious, and such misses will undermine public health efforts and put patients and their 
158 contacts at risk. This must give pause in the rush to approve additional testing options and 159 increase testing capacity, and emphasizes the importance of defining infectivity as a function of 160 viral load and other factors (e.g. time of exposure), which remains a critical unknown in this 161 pandemic.

162 Antigen detection assays promise rapid turnaround time, point-of-care implementation, and low 163 cost. For influenza detection, such tests have exhibited substantially lower analytical and clinical 164 sensitivity compared with NAAT tests (10). The poor historical performance for influenza 165 detection led to reclassification of influenza rapid antigen detection tests as Class II devices with 166 a new minimal performance standard of at least $80 \%$ sensitivity compared with NAAT (11).

167 Previously, clinical sensitivity of $50-88 \%$ for the Quidel Sofia influenza test was noted in several 168 studies in different influenza seasons compared to RT-PCR comparators (12-14). The same 169 trend was observed in our analysis of the single SARS-CoV-2 antigen test introduced thus far 170 with EUA status. Tests with such performance characteristics will identify individuals with the 171 highest viral burden. However, such a high detection threshold will be unlikely to fully meet 172 public or individual health goals in the COVID-19 pandemic.

173 Our findings also suggest that Ct values and imputed viral loads have clinical utility. Real-time 174 PCR methods in particular are inherently quantitative, and we demonstrate here that they are 175 quite reproducible during repeated clinical sampling over a short time period, with $R^{2}$ of 0.70 for 176 repeats within six hours (as a proxy for immediate repeats). We note that because PCR 177 efficiency can fall substantially with PCR cycle number, as we observed here, viral load is 178 ideally calculated not simply as a powers-of-2 transformation of Ct value but based on the 179 observed trend between efficiency and Ct number. This trend may differ by assay: for example, 180 the assay used here includes an internal control whose product may contribute to polymerase 181 inhibition. (This method can be extended to provide confidence limits that incorporate the 182 variance in, e.g., the Ct of the LoD, but this extension is beyond the scope of the current work.) 
183 As yet it is unclear whether or how viral loads affect prognosis, but they at least suggest a 184 measure of infectivity, as well as possibly severity of illness, and, therefore may have value for 185 public health efforts, as we learn which cutoffs may imply minimal or inconsequential infectivity, 186 especially during clearance of infection. We make explicit our assumption that $\sim 2$ virions per 187 reaction, translating to a viral load of 9 copies $/ \mathrm{mL}$, reflects a $100 \%$ detection rate. With stricter 188 cutoffs, clinical sensitivity falls slightly (e.g., from $90 \%$ to $86 \%$ for an assay with an LoD of 100 189 copies $/ \mathrm{mL}$, if using a cutoff of 4 copies $/ \mathrm{mL}$, or a single virion per reaction, and to $79 \%$ if using a 190 cutoff of 0.7 copies $/ \mathrm{mL}$, or a single virion per $3 \mathrm{~mL}$ transport tube). Regardless, these different 191 assumptions have essentially no effect on the relative clinical sensitivities of different assays. 192 While it is theoretically possible that even lower levels of infection are possible, making our 193 estimates of clinical sensitivity upper limits, we believe potential for contagion at these levels is 194 highly unlikely, as that would assume that breathing, a cough, or a sneeze would transmit more 195 particles than can be obtained by dedicated and vigorous physical swabbing of the actual nasopharynx.

197 To control the pandemic, ultimately we will need diagnostics for all three populations of interest, 198 infected, infectious, and susceptible, and for that we will need to understand whether and how 199 viral load relates to infectiousness. As we have shown, assays with higher LoD are likely to miss 200 non-negligible fractions of infected individuals. However, individuals with viral burdens low 201 enough to be missed by some assays may prove to be less infectious. In vitro, approximately 202 only 1 of 10,000 genome copies in viral cultures may be associated with a tissue culture 203 infectious viral particle based on standard preparation such as BEI Resources NR-52866(15). 204 However, it is unclear how or whether this fraction might change with viral load for patients in 205 vivo.

206 The ultimate lesson from these studies bears repetition: LoD matters and directly impacts efforts 207 to identify, control, and contain outbreaks during this pandemic. Various assays report out LoDs 
208 in manners that are often difficult to comprehend, for example, TCID $_{50}$ values that may related

209 to viral copy numbers in different ways depending on the viral preparation, or units of copies/ $\mu \mathrm{L}$

210 (1 copy $/ \mu \mathrm{L}=1,000$ copies $/ \mathrm{mL})$ or attomolar quantities $(1$ attomolar $=602 \mathrm{copies} / \mathrm{mL})$. We

211 therefore suggest that viral copies $/ \mathrm{mL}$ be used as a universal standard metric, so that cross

212 comparison between assays can readily be made. It is clear that viral load matters, and

213 therefore LoD values should be readily evaluable and in the public domain.

\section{Acknowledgements}

215 K.P.S. was supported by the National Institute of Allergy and Infectious Diseases of the National

216 Institutes of Health under award number F32 Al124590. The content is solely the responsibility

217 of the authors and does not necessarily represent the official views of the National Institutes of

218 Health. We would like to thank the clinical laboratory scientists and volunteers in the Beth Israel

219 Deaconess Medical Center microbiology laboratory for generating the data used in this 220 manuscript. 


\section{References}

223

224

225

226

227

228

229

230

231

232

233

234

235

236

237

238

239

240

241

242

243

244

245

246

1. Wang W, Xu Y, Gao R, Lu R, Han K, Wu G, Tan W. 2020. Detection of SARS-CoV-2 in Different Types of Clinical Specimens. Jama doi:10.1001/jama.2020.3786.

2. Lievens A, Van Aelst S, Van den Bulcke M, Goetghebeur E. 2012. Enhanced analysis of real-time PCR data by using a variable efficiency model: FPK-PCR. Nucleic Acids Res 40:e10.

3. Platts AE, Johnson GD, Linnemann AK, Krawetz SA. 2008. Real-time PCR quantification using a variable reaction efficiency model. Anal Biochem 380:315-22.

4. Smith KP, Cheng A, Chopelas A, DuBois-Coyne S, Mezghani I, Rodriguez S, Talay M, Kirby JE. 2020. Large-scale, in-house production of viral transport media to support SARS-CoV-2 PCR testing in a multi-hospital healthcare network during the COVID-19 pandemic. J Clin Microbiol doi:10.1128/jcm.00913-20.

5. Anonymous. Abbott RealTime SARS-CoV-2 Emergency Use Authorization Package Insert, REF 09N77-095, 51-608445/R1. Abbott Molecular Inc., Des Plaines, IL.

6. Shain EB, Clemens JM. 2008. A new method for robust quantitative and qualitative analysis of real-time PCR. Nucleic Acids Res 36:e91.

7. Callahan CJ, Lee R, Zulauf KE, Tamburello L, Smith KP, Previtera J, Cheng A, Green A, Azim AA, Yano A, Doraiswami N, Kirby JE, Arnaout RA. 2020. Open Development and Clinical Validation Of Multiple 3D-Printed Nasopharyngeal Collection Swabs: Rapid Resolution of a Critical COVID-19 Testing Bottleneck. J Clin Microbiol doi:10.1128/jcm.00876-20.

8. Anonymous. 2019-Novel Coronavirus (2019-nCoV) Real-Time RT-PCR Diagnostic Panel Emergency Use Authorization Instructions for Use, CDC-006-00019, Revision: 03 . CDC/DDID/NCIRD/ Division of Viral Disease, Atlanta, GA. 
247 9. Zhen W, Smith E, Manji R, Schron D, Berry GJ. 2020. Clinical Evaluation of Three Sample-To-Answer Platforms for the Detection of SARS-CoV-2. J Clin Microbiol doi:10.1128/jcm.00783-20.

10. Merckx J, Wali R, Schiller I, Caya C, Gore GC, Chartrand C, Dendukuri N, Papenburg J. 2017. Diagnostic Accuracy of Novel and Traditional Rapid Tests for Influenza Infection Compared With Reverse Transcriptase Polymerase Chain Reaction: A Systematic

254 11. Green DA, StGeorge K. 2018. Rapid Antigen Tests for Influenza: Rationale and Significance of the FDA Reclassification. J Clin Microbiol 56:e00711-18.

256 12. Arbefeville SS, Fickle AR, Ferrieri P. 2015. Sensitivity of the Quidel Sofia Fluorescent Immunoassay Compared With 2 Nucleic Acid Assays and Viral Culture to Detect

13. Kammerer PE, Radin JM, Hawksworth AW, Myers CA, Brice GT. 2016. Performance of the Quidel Sofia rapid influenza diagnostic test during the 2012-2013 and 2013-2014 influenza seasons. Influenza Other Respir Viruses 10:220-3.

262 14. Selove W, Rao LV. 2016. Performance of rapid SOFIA Influenza A+B test compared to 263 Luminex x-TAG respiratory viral panel assay in the diagnosis of influenza $A, B$, and 264 subtype H3. J Investig Med 64:905-7.

265 15. Anonymous. 2020. Certificate of Analysis for NR-52286. BEI Resources:Lot Numbers: 70033548 and 70034991. 
A

Repeats within 6 hours $(n=25)$

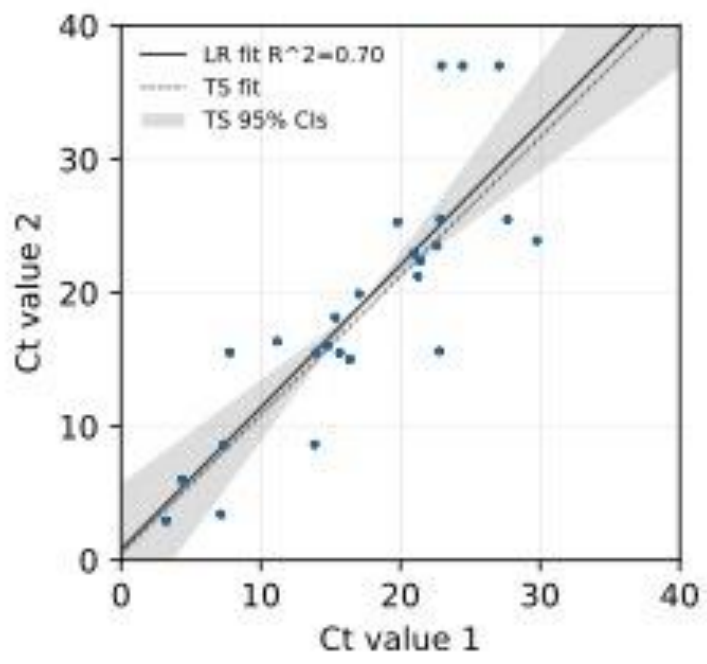

B Repeats within 12 hours $(n=51)$

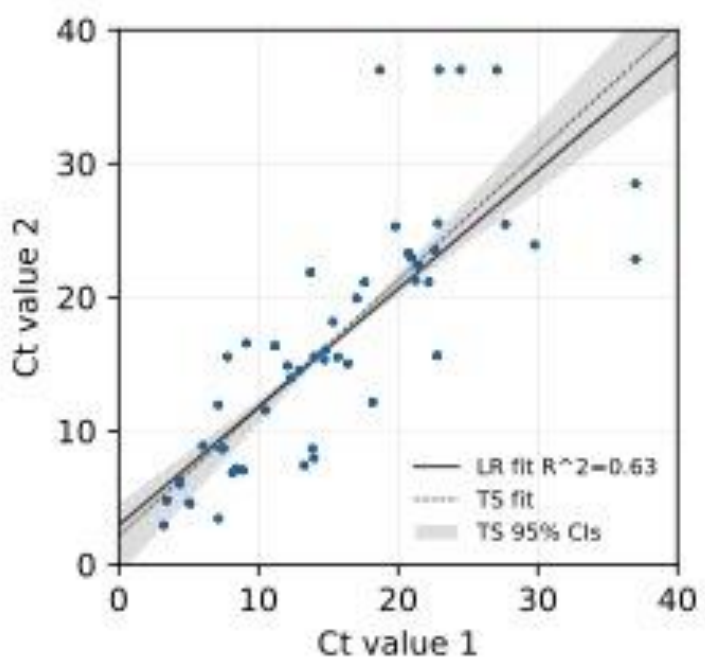

270 Figure 1: Ct values are highly repeatable. Data points shown are $\mathrm{Ct}$ values for SARS-CoV-2 271 testing of pairs of nasopharyngeal samples obtained within either 6 hours (A) or 12 hours (B) or 272 each other from the same patient, represented by the $X$ and $Y$ coordinates of each data point.

273 LR $=$ Linear Regression Fit. TS $=$ Theil-Sen Linear Regression Fit. Shade areas indicate $95 \%$ 274 confidence interval for TS fit. 


\section{A}

Binned histogram of viral loads $(n=4774)$

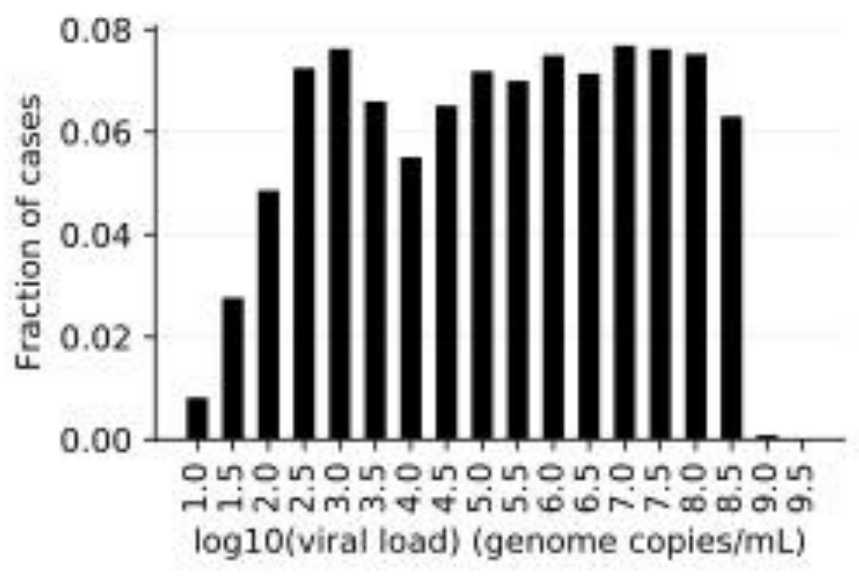

B

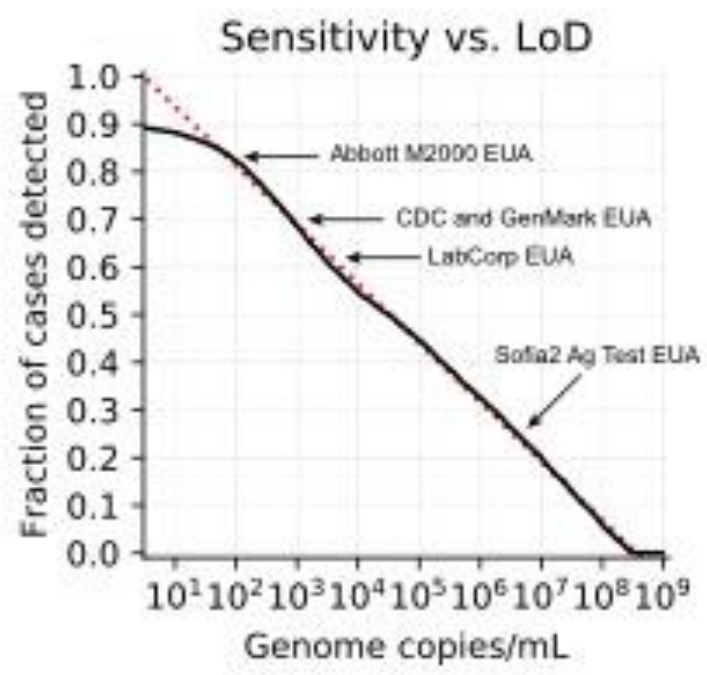

278 Figure 2: Viral load distribution and LoD. (A) Fraction of positive tests binned by $0.5 \log 10$ 279 bins of viral load. (B) Cumulative histogram distribution of viral loads showing percent detected 280 as a function of limit of detection - actual, solid line, and trend-line, dotted line. 
Repeats within 6 hours $(n=25)$

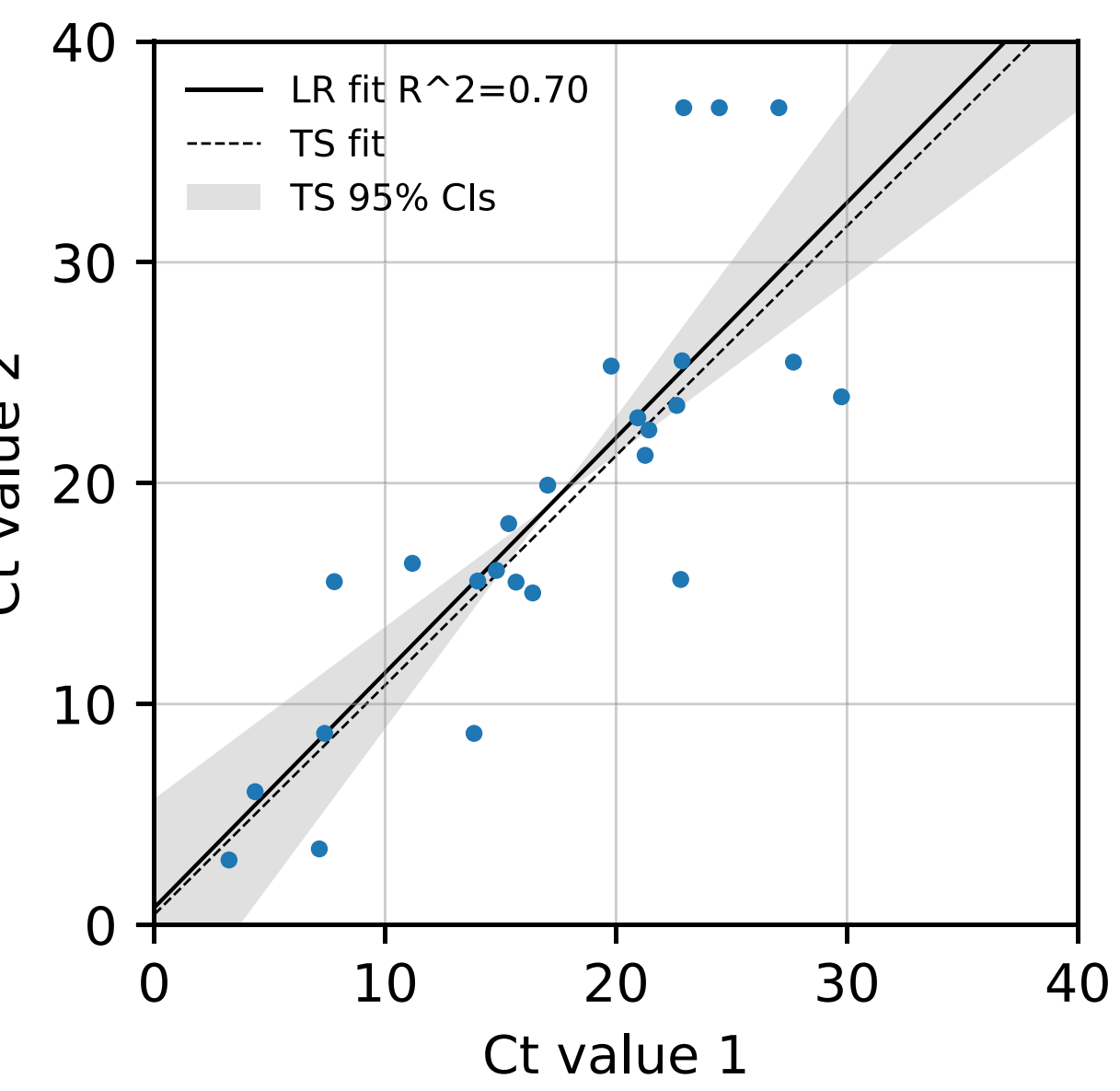

B

Repeats within 12 hours $(n=51)$

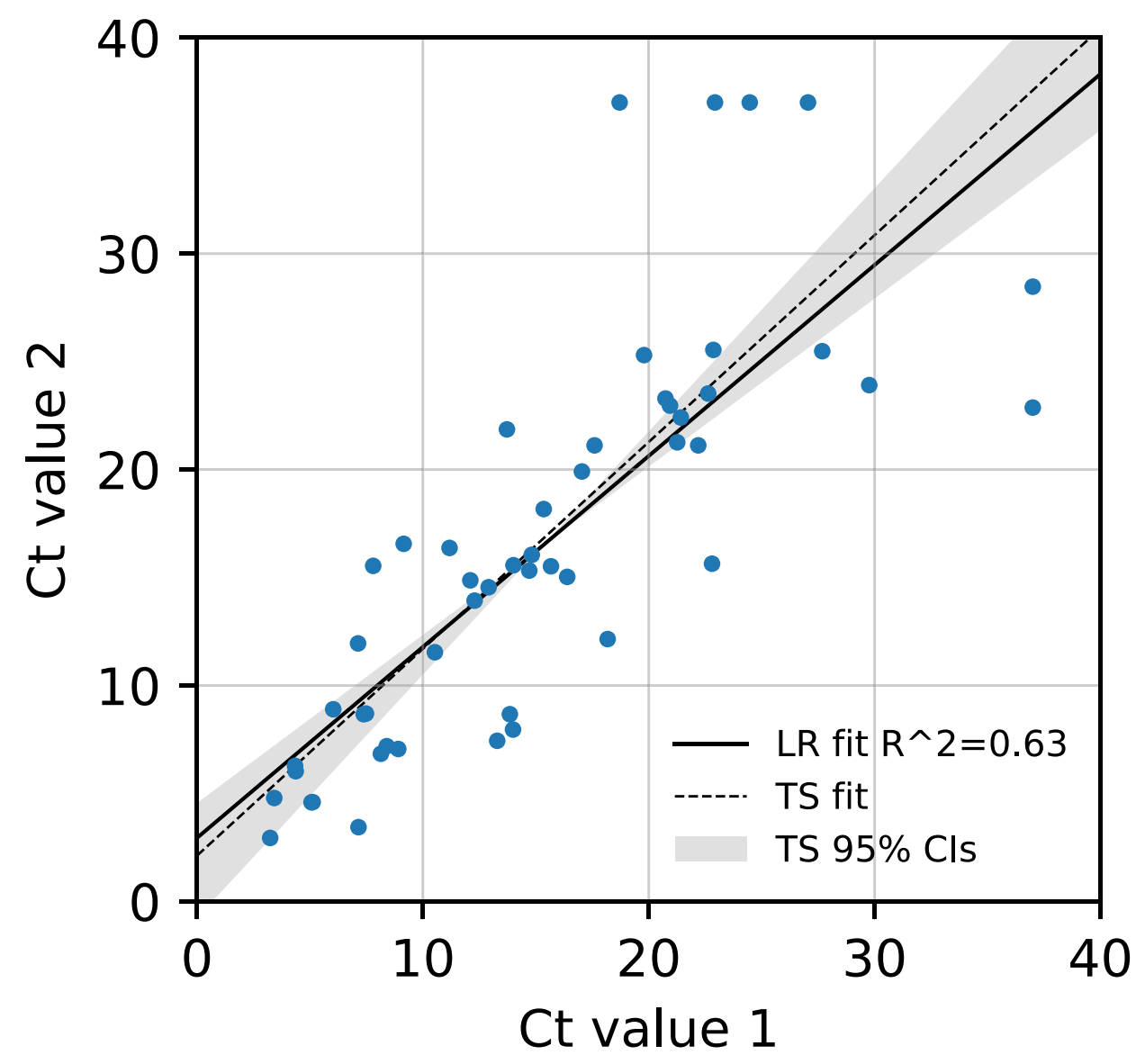


bioRx A preprint doi: https://doi.org/10.1101/2020.06.02.131144; this version posted June 4, 2020. The copyright holder for this preprint (which not certified by peer review) is the author/funder, who has granted bioRxiv a license to display the preprint in perpetuity. It is made available under aCC-BY-NC-ND 4.0 International license.

Binned histogram of viral loads $(n=4774)$

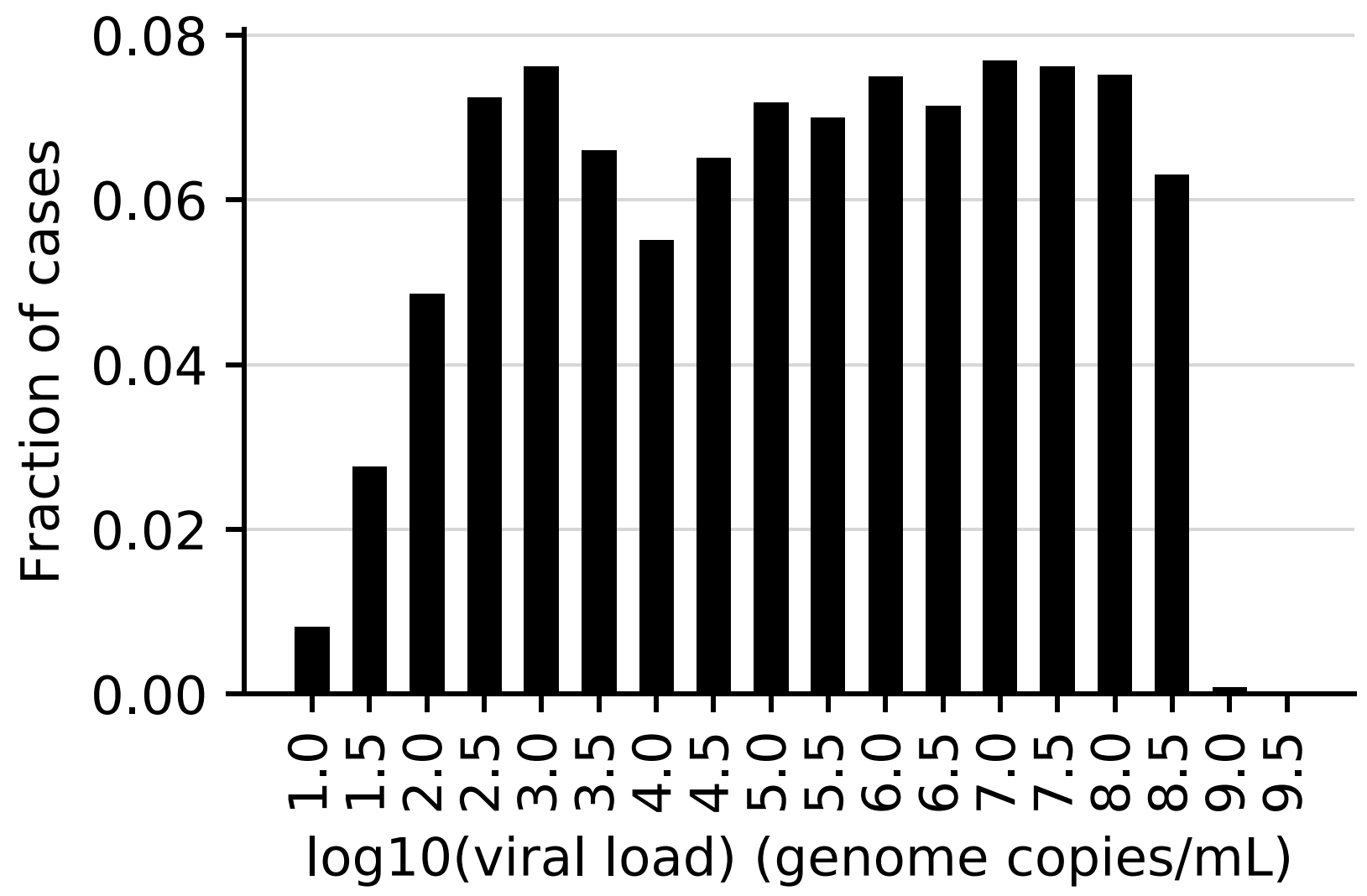

Sensitivity vs. LoD

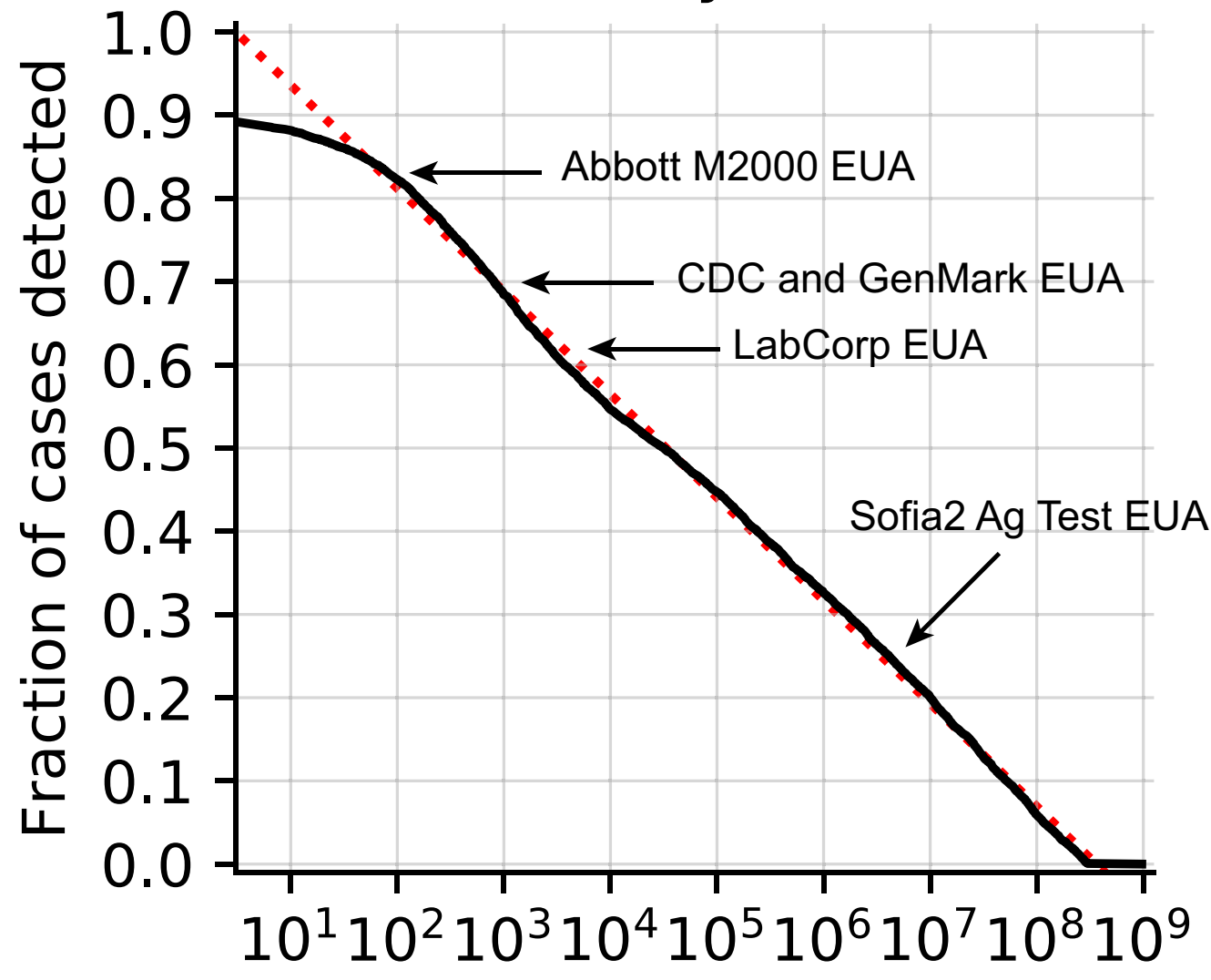

Genome copies/mL 